\title{
Erratum to: Crowdsourcing Versus the Laboratory: Towards Human-Centered Experiments Using the Crowd
}

\author{
Ujwal Gadiraju $^{1(凶)}$, Sebastian Möller ${ }^{2}$, Martin Nöllenburg ${ }^{3}$, \\ Dietmar Saupe ${ }^{4}$, Sebastian Egger-Lampl ${ }^{5}$, Daniel Archambault ${ }^{6}$, \\ and Brian Fisher ${ }^{7}$ \\ ${ }^{1}$ Leibniz Universität Hannover, Hannover, Germany \\ gadiraju@L3S.de \\ 2 TU Berlin, Berlin, Germany \\ 3 Algorithms and Complexity Group, TU Wien, Vienna, Austria \\ ${ }^{4}$ University of Konstanz, Konstanz, Germany \\ 5 Austrian Institute of Technology, Vienna, Austria \\ ${ }^{6}$ Swansea University, Swansea, UK \\ 7 Simon Fraser University, Burnaby, Canada
}

\section{Erratum to: \\ Chapter "Crowdsourcing Versus the Laboratory: Towards Human-Centered Experiments Using the Crowd" in: D. Archambault et al. (Eds.): Evaluation in the Crowd, LNCS 10264, https://doi.org/10.1007/978-3-319-66435-4_2}

The original version of this chapter contained an error in the third author's affiliation. The affiliation of Martin Nöllenburg was incorrect in the header of the paper. The author's affiliation has been corrected.

The updated online version of this chapter can be found at https://doi.org/10.1007/978-3-319-66435-4_2 